the body esteem domain increased from 48 to 81 . BAI scores reduced from 22.5 to 5 in 2 patients.

Conclusion Multidisciplinary assessment is important in selecting patients for bariatric surgery. This surgery should be performed in centres that can provide this. Improvements in quality of life are significant and important to monitor to sustain weight loss. Longer term follow-up is necessary to maintain weight loss and monitor progress.

\section{G99(P) GROUP EDUCATION IN ADOLESCENT DIABETES TRANSITION}

doi:10.1136/archdischild-2013-304107.111

'H Reid, ${ }^{2} \mathrm{~L}$ Potts, ${ }^{2} \mathrm{~K}$ Agostini, $2 \mathrm{~J}$ Luscombe, ${ }^{2 R}$ Thompson, 2, 3P Hindmarsh, 2, 3RM Viner, 2, ${ }^{3} \mathrm{~B}$ White. ${ }^{1}$ School of Medicine, University College London, London, UK; ${ }^{2}$ Children and Adolescent Diabetes Service, University College Hospital London, London, UK; ${ }^{3} / n s t i t u t e$ of Child Health, University College London, London, UK

Aims Education is a key component of the transition process. We piloted a half-day group education session for young people (YP) and their parents.

Methods All YP in the diabetes service aged 14-19 years were invited via letter, email (if address available) and/or telephone. Eligible participants were invited to book using a commercial web-based booking platform. Education was in the form of expert-delivered didactic group sessions and a parent workshop. Members of the adult diabetes team introduced their service and were available to meet families. Attendees completed evaluation forms at the end of the session.

Results Demographics: 25/130 (19\%) of eligible YP and 21 parents attended. 1/23 (4\%) eligible YP aged 14 years attended, 8/50 (16\%) aged 15-16 attended, 16/52 (28\%) aged 17-18 years attended and $1 / 5(20 \%)$ aged $19 y$ attended. 17/25 (68\%) YP in attendance were male. The mean (SD) HbA1c of attendees was $8.1 \%$ (1.4) vs $8.4 \%$ (1.7) for non-attendees ( $p, 0.05$, t-test). 24/25 (96\%) attendees had type 1 diabetes.

Booking and attendance: Of those invited by email: $21 / 34$ (62\%) of YP opened the email, 30/39 (77\%) of parents opened the email. The predominant barrier to attendance was exam commitments.

Acceptability and ratings: Mean (SD) YP rating for the session was $8.1(0.9)$ in a 10-point Likert scale (1 = very poor, $10=$ excellent). Mean (SD) parent ratings were 8.6 (1.4). After the session, $14 \%$ of YP reported they were "ready to move to adult services", $59 \%$ "more ready to move to adult services", $18 \%$ "equally ready" and $0 \%$ "less ready". Sessions most frequently rated by YP as "useful" were "rights and jobs" (77\%), "having a healthy baby" (64\%), "driving and diabetes" (59\%) and "alcohol and diabetes" (59\%); parents most rated "driving and diabetes" (94\%) "rights and jobs" (75\%), "adult clinic" (75\%) and "insulin pumps in adult services" (75\%).

Conclusions An expert-delivered group transition education session shows promise as an effective and easy to deliver tool for preparing adolescent patients for adult life with diabetes. Further exploratory work is needed to optimise this model and measure its effectiveness.

\section{G100(P) HYPERINSULINAEMIC HYPOGLYCAEMIA OF SHORT DURATION - CAN IT BE ASSOCIATED WITH SEVERE HYPOGLYCAEMIC BRAIN INJURY?}

doi:10.1136/archdischild-2013-304107.112

C Gilbert, K Morgan, L Hinchey, P Shah, K Hussain. Paediatric Endocrine Department (Hyperinsulinism), Great Ormond Street Hospital, London, UK

Background Neurological damage is a known risk associated with hyperinsulinaemic hypoglycaemia $(\mathrm{HH})$. Insulin suppresses ketone body formation and hence no alternative fuels are available for the brain to use; however it is not yet known how long HH has to last to cause brain injury. We report that neurological damage can occur after a short time in term, normal weight infants with diazoxide responsive $\mathrm{HH}$.

Aim To describe the clinical course and neurological outcome of 3 term neonates with severe hypoglycaemic brain injury who were not diagnosed with $\mathrm{HH}$ for at least 72 hours.

Methodology 3 patients who presented in the neonatal period with biochemically confirmed $\mathrm{HH}$ were recruited. Detailed clinical information was collected including MRI brain reports.

Results All three term neonates were discharged home after 24-36 hours of birth. Birth weight range was 2730-3460 gms and each delivery was classified as normal vaginal births with no associated risk factors for $\mathrm{HH}$. All infants presented to the Emergency department on day 3 to 4 of life with non-specific symptoms like poor feeding and lethargy. However all of them were noted to have jerky and seizure like movements. Biochemically, all had their true blood glucose levels less than $0.6 \mathrm{mmols} / \mathrm{L}$ with raised insulin and suppressed ketone body formation They all successfully responded to small doses $(5 \mathrm{mg} / \mathrm{kg} /$ day) of Diazoxide (two of them are off Diazoxide now and had transient hyperinsulinism). Each neonate had MRI brain due to clinical neurological concerns within the first few weeks of life that showed significant evidence of hypoglycaemic brain injury like gross white matter changes with parieto-occipital infarcts.

Conclusion It is very important for early identification and prompt management of $\mathrm{HH}$ as untreated severe hypoglycaemia can result in severe brain injury and subsequent neurodevelopmental handicap. Term infants with no risk factors are often difficult to identify due to non-specific symptoms. Parental education to recognise early symptoms of hypoglycaemia would be recommended and prompt medical advice should be sought. Blood glucose levels should be of utmost priority for babies presenting to $A \& E$ with non specific symptoms such as poor feeding/lethargy etc.

\section{G101(P) THE 0.12 FORMULA FOR THE MANAGEMENT OF HYPOGLYCAEMIA AND HYPERGLYCAEMIA IN CHILDREN WITH TYPE 1 DIABETES MELLITUS: VALIDATION AND SAFETY DATA}

doi:10.1136/archdischild-2013-304107.113

${ }^{1} \mathrm{~L}$ Yazbeck, ${ }^{2} \mathrm{M}$ Watson, ${ }^{2} \mathrm{~N}$ Ninis, ${ }^{2} \mathrm{~S}$ Wassouf. 'Clinical Genetics, Kennedy Galton Centre, London, UK; 'Paediatrics, Imperial College NHS trust, London, UK

Background The life of patients with diabetes mellitus is populated with hypo- and hyperglycaemias, both of which are associated with inherent dangers.

Existing formulas attempting to quantify patients' insulin requirements have proved ineffective and rather arbitrary ${ }^{1}$; they are based on estimations of 24 hour consumptions of insulin and carbohydrates in the average person.

Objective This paper tests the effectiveness of the 0.12 formula that is based on patient's weight and carbohydrates consumption. It calculates glucose and insulin sensitivity and guides the treatment of hypo- and hyperglycaemia, specifically for each patient. Method Data from the Continuous Glucose monitoring system (CGMS) applications and the associated food diary were used to assess the blood sugar achieved after hypo- and hyperglycaemia treatment. This was compared to that expected via the 0.12 formula using the Wilcoxon statistical analysis.

Results 20 and 42 patients were assessed for hypoglycaemia and hyperglycaemia respectively.

In either treatment, there was no statistically significant difference between expected and achieved blood sugar; $\mathrm{p}$-values were 0.53 and 0.072 respectively.

Furthermore, insulin sensitivity derived using the 0.12 formula was compared to that calculated through the historically popular 100 rule $^{1}$. Wilcoxon statistical analysis showed significant statistical difference between the two formulas; p-value 0.0025, (confidence interval $+/-0.000484$ ). 